\title{
Creep calculation for a three-layer beam with a lightweight filler
}

\author{
Viacheslav Chepurnenko, ${ }^{1, *}$, Batyr Yazyev ${ }^{1}$, and Xuanzhen Song ${ }^{1}$ \\ ${ }^{1}$ Don State Technical University, 344022 Sotsialisticheskaya 162, Russia
}

\begin{abstract}
In the article the technique of calculation of a three-layer beam with a lightweight filler taking into account the creep of the middle layer is given. The problem reduces to a second-order differential equation, which is solved numerically by the method of finite differences. An example of a calculation is presented for a hinged at the ends beam under the action of a uniformly distributed load. The linear Maxwell-Thompson equation is used as the creep law. Solution was performed in software package Matlab.
\end{abstract}

\section{Introduction}

Products containing polymers are more prone to creep than many other building materials. Since the middle layer of three-layer panels is often made of foams, this property must be taken into account in the design. The consideration of creep is associated with the solution of complex systems of integro-differential equations [1-7], which significantly hinders the use of the apparatus of creep theory in the practice of engineering calculations. In this paper, a comparatively simple method for calculating a three-layer beam considering creep is given, which can be used in engineering practice.

\section{Formulation of the problem}

We consider a hinged-supported at the ends beam, loaded with a uniformly distributed load $q$ (Fig. 1).

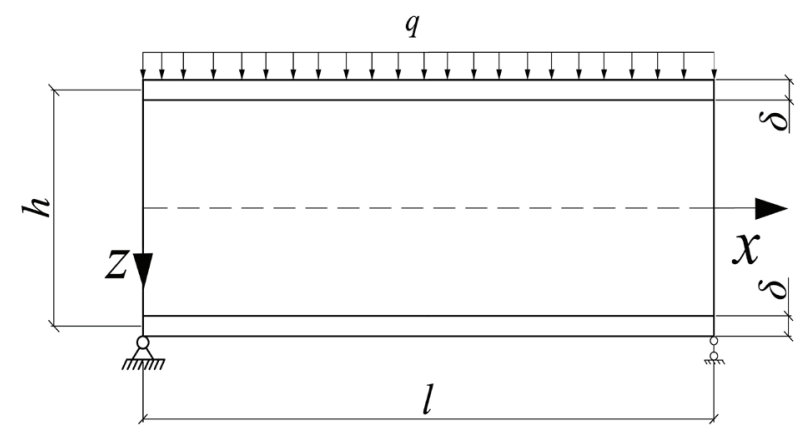

Fig. 1. Calculation scheme.

In deriving the equations, the following hypotheses are accepted:

1 . The bending moment is completely perceived by the outer layers.

Proceeding from this hypothesis, the bending moment is related to the stresses in the upper and lower sheathing as follows:

$$
M(x)=\sigma^{+} b \delta \frac{h}{2}-\sigma^{-} b \delta \frac{h}{2},
$$

where $b$ - beam width, $h$ - beam height, $\delta$ - thickness of the sheathings, $\sigma^{+}-$stress in the bottom sheathing, $\sigma^{-}-$stress in the upper sheathing.

Expression (1) can be rewritten as:

$$
\frac{M(x)}{I}=\frac{\sigma^{+}-\sigma^{-}}{h},
$$

where $I=b \delta \frac{h^{2}}{2}-$ moment of inertia of the sheathings.

2. The displacements along the thickness of the filler are distributed according to a linear law:

$$
u_{m}(z)=\frac{u^{+}+u^{-}}{2}+\frac{u^{+}-u^{-}}{h} z
$$

where $u^{+}$and $u^{-}-$respectively displacements of the lower and upper skin, $u_{m}$ - displacement of the middle layer.

3. The transverse force is completely perceived by the filler, the filler works only on shear, and the tangential stresses along its thickness are constant. Proceeding from this hypothesis, the transverse force is defined as follows:

$$
Q=\tau_{m} b h=G_{m} \gamma_{m}^{e l} b h,
$$

where $\tau_{m}-$ shear stress in the filler, $G_{m}-$ shear modulus of the filler, $\gamma_{m}^{e l}$ - elastic shear deformation of the filler, representing the difference between total shear deformation and creep deformation:

$$
\gamma_{m}^{e l}=\gamma_{m}-\gamma_{m}^{*}
$$

\footnotetext{
${ }^{*}$ Corresponding author: anton chepurnenk@mail.ru
} 


\section{The derivation of the resolving equations}

The stresses in the sheathings are related to the displacements as follows:

$$
\sigma^{+}=E \frac{\partial u^{+}}{\partial x} ; \quad \sigma^{-}=E \frac{\partial u^{-}}{\partial x}
$$

where $E-$ modulus of elasticity of sheathings.

Substituting (5) into (2), we obtain:

$$
\frac{M}{E I}=\frac{1}{h} \frac{\partial}{\partial x}\left(u^{+}-u^{-}\right)
$$

The displacement and deformation of the filler are related by the Cauchy equation:

$$
\gamma_{m}=\frac{\partial u_{m}}{\partial z}+\frac{\partial w}{\partial x}=\frac{u^{+}-u^{-}}{h}+\frac{\partial w}{\partial x} .
$$

We substitute (8) in (5), and then (5) in (4):

$$
Q=G_{m} b h\left(\frac{u^{+}-u^{-}}{h}+\frac{\partial w}{\partial x}-\gamma_{m}^{*}\right) .
$$

According to Zhuravskii's theorem, the transverse force is related to the intensity of the uniformly distributed load as follows:

$$
\frac{\partial Q}{\partial x}=-q
$$

Differentiating (9) with respect to $x$, we obtain:

$$
G_{m} b h\left(\frac{1}{h} \frac{\partial}{\partial x}\left(u^{+}-u^{-}\right)+\frac{\partial^{2} w}{\partial x^{2}}-\frac{\partial \gamma_{m}^{*}}{\partial x}\right)=-q
$$

Substituting (7) into (10), we obtain the basic resolving equation:

$$
\frac{\partial^{2} w}{\partial x^{2}}=-\frac{M(x)}{E I}-\frac{q}{G_{m} b h}+\frac{\partial \gamma_{m}^{*}}{\partial x} .
$$

\section{Method of solving the problem}

Equation (11) is solved numerically by the method of finite differences. The bending moment and shear force for considered beam are determined as follows:

$$
M(x)=\frac{q x}{2}(l-x) ; \quad Q(x)=\frac{q l}{2}-q x .
$$

The boundary conditions have the form:

$$
w_{x=0}=w_{x=l}=0
$$

The time interval is divided into $n_{t}$ steps $\Delta t$. At the first stage we perform the solution of the elastic problem $\left(\gamma_{m}^{*}=0\right)$. A uniform grid in $x$ with step $\Delta x$ is introduced. The second derivative of the deflection in the node with the number $i$ using central differences is written in the form:

$$
\left.\frac{\partial^{2} w}{\partial x^{2}}\right|_{i}=\frac{w_{i+1}-2 w_{i}+w_{i-1}}{\Delta x^{2}} .
$$

As a result, the problem reduces to a system of linear algebraic equations:

$$
[A] \cdot\{X\}=\{B\}
$$

where $[A] \quad-$ matrix of coefficients, $\{X\}=\left\{w_{1} w_{2} \ldots w_{n+1}\right\}^{T}-$ vector of unknown nodal displacements, $\{B\}=\left\{0 f_{2} f_{3} \ldots f_{n} 0\right\}^{T}-$ vector of free terms.

The coefficients $f_{i}$ are defined as follows:

$$
f_{i}=-\frac{M_{i}}{E I}-\frac{q}{G_{m} b h}+\left.\frac{\partial \gamma_{m}^{*}}{\partial x}\right|_{i}
$$

Matrix $[A]$ has the form:

$$
[A]=\left[\begin{array}{cccccccc}
1 & 0 & 0 & 0 & \ldots & 0 & 0 & 0 \\
a_{2} & b_{2} & c_{2} & 0 & \ldots & 0 & 0 & 0 \\
0 & a_{3} & b_{3} & c_{3} & \ldots & 0 & 0 & 0 \\
\ldots & \ldots & \ldots & \ldots & \ldots & \ldots & \ldots & \ldots \\
0 & 0 & 0 & 0 & \ldots & a_{n} & b_{n} & c_{n} \\
0 & 0 & 0 & 0 & \ldots & 0 & 0 & 1
\end{array}\right],
$$

where $a_{i}=c_{i}=\frac{1}{\Delta x^{2}}, b_{i}=-\frac{2}{\Delta x^{2}}$.

It follows from formula (4) that for a statically determinate beam, the tangential stresses do not change during the creep process.

If the creep law is given in differential form, then knowing the creep strain and stress at the current time, we can determine the rate of growth of the creep strain and its magnitude at the time $t+\Delta t$ :

$$
\gamma_{m}^{*}(t+\Delta t)=\gamma_{m}^{*}(t)+\frac{\partial \gamma_{m}^{*}}{\partial t} \Delta t
$$

Numerical differentiation of the function $\gamma_{m}^{*}$ with respect to $x$ is performed by the formula:

$$
\left.\frac{\partial \gamma_{m}^{*}}{\partial x}\right|_{i}=\frac{\gamma_{m, i+1}^{*}-\gamma_{m, i-1}^{*}}{2 \Delta x}
$$

The formula (12) is applicable only for internal grid nodes. For the first and last node, it is not necessary to calculate the derivative $\frac{\partial \gamma_{m}^{*}}{\partial x}$. 


\section{Results and discussion}

The test task was solved with the following initial data: $l=1.5 \mathrm{~m}, b=0.1 \mathrm{~m}, h=0.06 \mathrm{~m}, q=0.82 \mathrm{kN} / \mathrm{m}$, $G_{m}=25 \mathrm{MPa}, E=0.71 \cdot 10^{5} \mathrm{MPa}, \delta=1 \mathrm{~mm}$.

We used the deformation law, which is described by the Maxwell-Thompson equation [8]:

$$
\kappa \frac{\partial \gamma_{m}}{\partial t}+H \gamma_{m}=n \frac{\partial \tau_{m}}{\partial t}+\tau_{m}
$$

where $\kappa=n G_{m}=56 \mathrm{MPa} \cdot$ day $\quad-$ coefficient of viscosity of the filler, $n$ - time of relaxation, $H=15 \mathrm{MPa}-$ long shear modulus of the filler.

The total deformation is represented as the sum of elastic deformation and creep deformation:

$$
\gamma_{m}=\frac{\tau_{m}}{G_{m}}+\gamma_{m}^{*}
$$

Substituting (14) into (13), we obtain an expression for the growth rate of the creep strain:

$$
\frac{\partial \gamma_{m}^{*}}{\partial t}=\frac{1}{\kappa}\left[\left(1-\frac{H}{G_{m}}\right) \tau_{m}-H \gamma_{m}^{*}\right]
$$

The resulting curve of the deflection growth in the middle of the span is shown in Fig. 2.

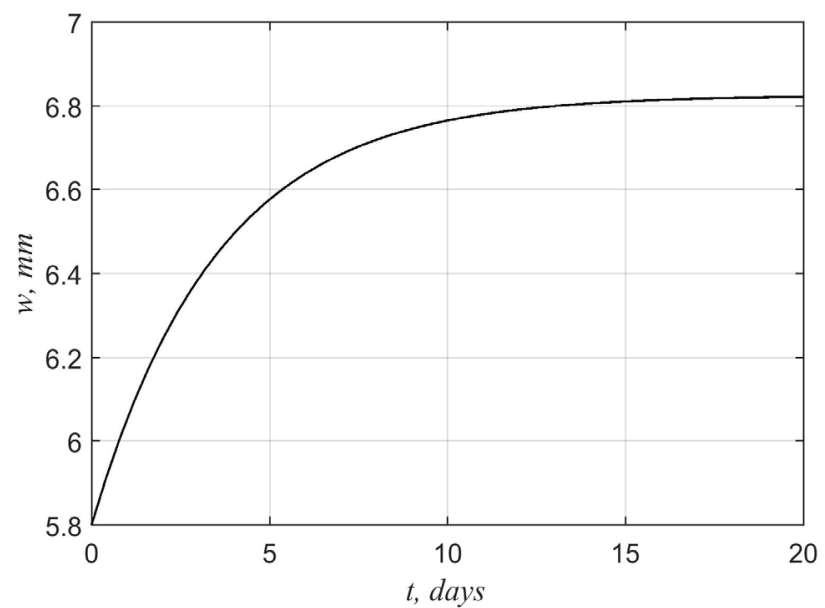

Fig. 2. The graph of the deflection growth

We note that under the law of creep (15), the problem can be solved analytically. The law of creep (15) can be rewritten in the form:

$$
\frac{\partial \gamma_{m}^{*}}{\partial t}=k_{1}-k_{2} \gamma_{m}^{*}
$$

where $k_{1}=\frac{\left(1-\frac{H}{G_{m}}\right) \tau_{m}}{n G_{m}}, \quad k_{2}=\frac{H}{n G_{m}}$.

We divide the variables in Eq. (16):

$$
\frac{\partial \gamma_{m}^{*}}{k_{1}-k_{2} \gamma_{m}^{*}}=\partial t
$$

Integrating the expression (17), we obtain:

$$
-\frac{1}{k_{2}} \ln \left|k_{1}-k_{2} \gamma_{m}^{*}\right|=t+\ln C(x)
$$

where $C(x)$ - arbitrary function of integration which is determined from condition:

$$
\left.\gamma_{m}^{*}\right|_{t=0}=0 \rightarrow-\frac{1}{k_{2}} \ln \left|k_{1}\right|=\ln C(x) \rightarrow C(x)=k_{1}^{-1 / k_{2}} .
$$
form:

The dependence of the creep strain on time takes the

$$
\gamma_{m}^{*}(t)=\frac{k_{1}}{k_{2}}\left(1-e^{-k_{2} t}\right)=\tau_{m}\left(\frac{1}{H}-\frac{1}{G_{m}}\right)\left(1-e^{-\frac{H}{n G_{m}} t}\right)
$$

Differentiating expression (18) with respect to $x$, we obtain:

$$
\begin{aligned}
& \frac{\partial \gamma_{m}^{*}}{\partial x}=\frac{\partial \tau_{m}}{\partial x}\left(\frac{1}{H}-\frac{1}{G_{m}}\right)\left(1-e^{-\frac{H}{n G_{m}} t}\right)= \\
& =\frac{1}{b h} \frac{\partial Q}{\partial x}\left(\frac{1}{H}-\frac{1}{G_{m}}\right)\left(1-e^{-\frac{H}{n G_{m}} t}\right)= \\
& =-\frac{q}{b h}\left(\frac{1}{H}-\frac{1}{G_{m}}\right)\left(1-e^{-\frac{H}{n G_{m}} t}\right) .
\end{aligned}
$$

Equation (11) for considered beam takes the form:

$$
\frac{\partial^{2} w}{\partial x^{2}}=-\frac{1}{E I} \frac{q x}{2}(l-x)-\frac{q}{G(t) A},
$$

where $\frac{1}{G(t)}=\frac{1}{G_{m}}+\left(\frac{1}{H}-\frac{1}{G_{m}}\right)\left(1-e^{-\frac{H}{n G_{m}} t}\right), \quad A=b h$.

Integrating equation (19) with respect to $x$, we obtain:

$$
\begin{gathered}
\frac{\partial w}{\partial x}=-\frac{1}{E I}\left(\frac{q x^{2} l}{4}-\frac{q x^{3}}{6}\right)-\frac{q}{G(t) A} x+C_{1}(t) . \\
w(x)=-\frac{1}{E I}\left(\frac{q x^{3} l}{12}-\frac{q x^{4}}{24}\right)-\frac{q}{G(t) A} \frac{x^{2}}{2}+C_{1}(t) x+C_{2}(t) .
\end{gathered}
$$

Functions $C_{1}(t)$ and $C_{2}(t)$ we determine from the boundary conditions: 


$$
\begin{aligned}
& \left.w\right|_{x=0}=0 \rightarrow C_{2}(t)=0 ; \\
& \left.w\right|_{x=l}=0:-\frac{1}{E I}\left(\frac{q l^{4}}{12}-\frac{q l^{4}}{24}\right)-\frac{q}{G(t) A} \frac{l^{2}}{2}+C_{1}(t) l=0 \rightarrow \\
& \rightarrow C_{1}(t)=\frac{q l}{2 G(t) A}+\frac{q l^{3}}{24 E I} .
\end{aligned}
$$

The expression for the deflection in the middle of the span takes the form:

$$
\left.w\right|_{x=\frac{l}{2}}=\frac{5 q l^{4}}{384 E I}+\frac{q l^{2}}{8 G(t) A} .
$$

At $t=0$, the maximum deflection is defined as

$$
\left.w_{\max }\right|_{t=0}=\frac{5 q l^{4}}{384 E I}+\frac{q l^{2}}{8 G_{m} A} .
$$

Furthermore, at $t \rightarrow \infty$ substitution in the formula (20) gives:

$$
\left.w_{\max }\right|_{t \rightarrow \infty}=\frac{5 q l^{4}}{384 E I}+\frac{q l^{2}}{8 H A} .
$$

Substituting the initial data into the formulas (21) and (22), we obtain at $t=0 w_{\max }=5.77 \mathrm{~mm}$ and at $t \rightarrow \infty w_{\max }=6.79 \mathrm{~mm}$. The results obtained numerically almost coincide with the analytical solution.

\section{Summary}

When solving a test problem, one of the simplest creep laws was considered, which is valid for linearly viscoelastic material. However, Eq. (11) allows to specify an arbitrary creep law, including a nonlinear one. For polymers, the nonlinear Maxwell-Gurevich equation [9] agrees well with the experimental data. The method for determining the relaxation constants included in this equation is given in [9-10].

It is necessary to note that an analogous problem was solved in [11].The Voigt law was used, which has the form:

$$
\tau_{m}=\kappa \frac{\partial \gamma_{m}}{\partial t}+H \gamma_{m}
$$

The authors of the paper [11] have reduced the problem to a system of two differential equations, which is not very convenient.

If the stress $\tau_{m}$ is constant, then the derivative $\frac{\partial \tau_{m}}{\partial t}$ vanishes and the results obtained on the basis of the Maxwell-Thompson law must coincide with the results based on the Voigt law.

Comparison of our results with work [11] is not possible, since in this paper the authors do not give all the initial data necessary for calculating.
An experimental study of the creep of foam filler and three-layer beams is carried out in [12-13]. However, the authors use Findley's power law, in which the time is contained in an explicit form. Such a law cannot be used for complex loading regimes.

Thus, the question of approximating the creep curves of the filler on the basis of the Maxwell-Gurevich equation remains valid.

\section{References}

1. S. Litvinov, L. Trush, S. Yazyev, Procedia Engineering, 150, pp. 1686-1693 (2016)

2. S. Litvinov, E. Klimenko, I. Kulinich, S. Yazyeva, International Polymer Science and Technology, 2, pp. 23-25 (2015).

3. V. Andreev, A. Avershyev, WIT Transactions on the Built Environment. Fluid Structure Interaction VII , pp. 123-132 (2013).

4. V. Andreev, E. Barmenkova, Applied Mechanics and Materials, 204-208, pp. 3596-3599 (2012)

5. V. Andreev, A. Chepurnenko, B. Yazyev, Advanced Materials Research, 1004-1005, pp. 257-260 (2014)

6. S. Litvinov, L. Trush, A. Dudnik, Engineering Bulletin of the Don, 2, 2016. URL: http://www.ivdon.ru/en/magazine/archive/n2y2016/ $\underline{3560 .}$.

7. V. Andreev, B. Yazyev, A. Chepurnenko, Advanced Materials Research, 900, pp. 707-710 (2014)

8. A. Chepurnenko, L. Mailyan, B. Jazyev, Procedia Engineering, 165, pp. 990 - 994 (2016)

9. A. Chepurnenko, V. Andreev, A. Beskopylny, B. Jazyev, MATEC Web of Conferences, 06059 (2016). URL: http://www.matecconferences.org/articles/matecconf/pdf/2016/30/mat ecconf smae2016 06059.pdf

10. A. Dudnik, A. Chepurnenko, S. Litvinov, Plastic masses, 1-2, pp. 30-33 (2016)

11. A. Gavrilov, Yu. Osetinsky, Light construction of building coverings, pp. 76-82 (1976).

12. M. Garrido, J. Correia, F. Branco, T. Keller, Journal of Composite Materials, 48(18), pp. 2237-2249 (2014).

13. M. Sa et al. Composite Structures, 93, pp. 24092418 (2011) 